\title{
The distribution of the ISM in the Milky Way
}

\section{A three-dimensional large-scale model}

\author{
A. Misiriotis ${ }^{1}$, E. M. Xilouris ${ }^{2}$, J. Papamastorakis ${ }^{1}$, P. Boumis ${ }^{2}$, and C. D. Goudis ${ }^{2,3}$ \\ ${ }^{1}$ University of Crete, Physics Department, PO Box 2208, 71003 Heraklion, Crete, Greece \\ 2 National Observatory of Athens, I. Metaxa \& Vas. Pavlou Str., Palaia Penteli, 15236 Athens, Greece \\ 3 Astronomical Laboratory, Department of Physics, University of Patras, 26110 Patras, Greece \\ Received 1 December 2005 / Accepted 4 July 2006
}

\section{ABSTRACT}

\begin{abstract}
We use the COBE/DIRBE $(1.2,2.2,60,100,140$, and $240 \mu \mathrm{m})$ maps and the COBE/FIRAS spectra (for the wavelength range $100-1000 \mu \mathrm{m})$ to constrain a model for the spatial distribution of the dust, the stars, and the gas in the Milky Way. By assuming exponential axisymmetric distributions for the dust and the stars and by performing the corresponding radiative transfer calculations we closely (given the simple geometry of the model) reproduce the FIR and NIR maps of the Milky Way. Similar distributions for the atomic and molecular hydrogen in the disk are used (with an inner cut-off radius for the atomic hydrogen) to fit the gas data. The star formation rate as a function of the Galactic radius is derived from the FIR emission and is well in agreement with existing estimates from various star formation tracers. The gas surface density is plotted against the star formation rate density and an "intrinsic" Galactic Schmidt law is derived with excellent agreement with the "external" Schmidt law found for spiral galaxies. The Milky Way is found to consume $\sim 1 \%$ and $\sim 10 \%$ of its gas in the outer and inner regions respectively (for a period of $0.1 \mathrm{Gyr}$ ) to make stars. The dust-induced $B-V$ color excess observed in various directions and distances (up to $\sim 6.5 \mathrm{kpc}$ ) with well-studied Cepheid stars is compared with the model predictions showing a good agreement. The simple assumption of exponential distributions of stars and dust in the Galaxy is found to be quite instructive and adequate in modeling all the available data sets from $0.45 \mu \mathrm{m}$ ( $B$-band) to $1000 \mu \mathrm{m}$.
\end{abstract}

Key words. dust, extinction - ISM: structure - Galaxy: structure

\section{Introduction}

Our Galaxy, the Milky Way, constitutes the best laboratory for studying the properties of the interstellar medium (ISM) in spiral galaxies. Being inside the Galaxy, the observer has the advantage of looking through different lines of sight passing both through dense environments (e.g., the Galactic center) and through areas almost free of dust and gas (e.g., the Galactic poles). The way that dust and gas (both in molecular and neutral phases) are distributed inside the Galaxy not only shapes the appearance of the Galaxy in different wavelengths, but also drives star formation, a key process that determines Galactic evolution.

There are several tracers for measuring the star formation activity in a galaxy (e.g., ultra-violet (UV) emission, far-infrared (FIR) emission, $\mathrm{H}_{\alpha}$, and radio emission), yet it is far from trivial to get an accurate estimate (see Kennicutt 1998b, for a review). Most of the star formation rate (SFR) tracers are affected by dust extinction, thus rendering their use quite limited and uncertain (see, however, Kewley et al. 2002, for a discussion on properly accounting for extinction). The far infrared (FIR) emission, if modeled properly to distinguish between the diffuse dust emission and the emission coming from the star formation complexes, has been proven to be one of the most promising SFR indicators (see Misiriotis et al. 2004; and for a review Kylafis \& Misiriotis 2005).

Some efforts have been made in modeling the interstellar medium (ISM) in the Milky Way. Kent et al. (1991) used the $K$-band data from the SPACELAB infrared telescope and modeled the distribution of the light in the Galaxy by assuming an exponential distribution for the starlight in the disk and the bulge. Using the COBE 140 and $240 \mu \mathrm{m}$ maps along with radio surveys of $\mathrm{HI}$ and $\mathrm{H}_{2}$, Sodroski et al. (1994) modeled the FIR emission of the Galaxy and derived the dust temperature profiles as well as gas-to-dust mass ratios and emissivities along different lines of sight. A composite of COBE/DIRBE and IRAS/ISSA maps made by Schlegel et al. (1998) provides a unique tool for estimating the Galactic extinction along different directions. Davies et al. (1997) modeled the FIR emission from the Galaxy by fitting exponential dust distributions to the COBE/DIRBE maps. In this study, the authors find that the best fit could be achieved by assuming that the dust distribution is more extended than that of the stars, namely that the scalelength of the dust is 1.5 times that of the stars and the scaleheight of the dust is twice that of the stars. Modeling of nearby edge-on galaxies (e.g., Xilouris et al. 1999, and references therein) do support the finding of the dust having a larger scalelength with respect to the stellar one, but not the scaleheight relation found in this study. In our analysis we will perform modeling of the near-infrared (NIR) and the FIR maps of the Milky Way and thus we will investigate the relation between the dust and stellar geometrical characteristics. Drimmel \& Spergel (2001) presented a very detailed modeling of the Milky Way dust content. Based on a total of 48 parameters (26 parameters for the dust and 22 parameters for the stars), this model quite nicely fitted the NIR ( $J$-band) and FIR $(240 \mu \mathrm{m})$ radial profiles. In a subsequent paper (Drimmel et al. 2003), this model was used to calculate the extinction to any point within the Galactic disk. This model provides a detailed description of the Galactic morphology, taking into account the structures of the disk, the spiral arms, and the warp. In contrast, our approach aims to model the Galaxy using as few parameters as possible. We know in advance that we will not be able to get an excellent representation of the detailed morphology, but we will be able to 
draw results on the large-scale distribution of the dust, the stars, and the star formation in our Galaxy.

In this paper we use a simple (with the minimal number of parameters required), but realistic, three-dimensional model, which includes the effects of absorption and scattering of the stellar light by the dust in the optical and near infrared (NIR) wavelengths. We also take into account the emission from the diffuse dust and the emission from a warm dust component associated with the star-forming regions. In order to constrain the model parameters, we fit the model to all the available data simultaneously. The data that we use consist of the COBE/DIRBE $(1.2,2.2,60,100,140$, and $240 \mu \mathrm{m})$ maps and the COBE/FIRAS spectra (for the wavelength coverage between 100 and $10000 \mu \mathrm{m}$ ). Using published maps for the atomic and molecular hydrogen, we model the gas distribution. After calculating the SFR density throughout the Galactic disk and comparing it with the observed gas surface density, we investigate the validity of the Schmidt (1959) law as quantified by Kennicutt (1998a). Finally, using a catalog of Cepheid stars with good estimates of their distance and $B-V$ color excess, we confirm the validity of our model in the optical region.

\section{The data}

\subsection{The COBE data}

The Cosmic Background Explorer (COBE) satellite, launched on November 18, 1989, has been proved to be a very successful space mission (Boggess et al. 1992). With the Diffuse Infrared Background Experiment (DIRBE) instrument, COBE surveyed the entire sky in 10 photometric bands covering the wavelength region from 1 to $240 \mu \mathrm{m}$ at an angular resolution of 0.7 square degrees (Sodroski et al. 1994). Using another instrument, the Far Infrared Absolute Spectrometer (FIRAS), COBE measured the spectrum of the dust emission of our Galaxy in the wavelength range from $120 \mu \mathrm{m}$ to $10000 \mu \mathrm{m}$ (Fixsen et al. 1996). These two instruments provide us with a unique set of data of the dust emission for a wide range of wavelengths, ideal for studying the properties and the distribution of dust in the Galaxy.

\subsubsection{NIR and FIR maps}

DIRBE was designed primarily to conduct a systematic search for an isotropic cosmic infrared background in 10 photometric bands from 1.25 to $240 \mu \mathrm{m}$ (Boggess et al. 1992; Hauser et al. 1998). Boggess et al. (1992) report an rms sensitivity per field of view (0.7 square degrees) of 1, 0.9, 0.4, 0.1, 11, and $4\left(10^{9} \mathrm{~W} \mathrm{~m}^{-2} \mathrm{sr}^{-1}\right)$ at $1.2,2.2,60,100,140$, and $240 \mu \mathrm{m}$, respectively.

For the purposes of this study we make use of the 1.2, 2.2, 60, 100, 140, and $240 \mu \mathrm{m}$ Zodi-Subtracted Mission Average maps obtained from the COBE database. This set of data consists of weekly averaged intensity maps with the zodiac light subtracted from the maps (see Kelsall et al. 1998 for a description of the model for the interplanetary dust). The data were extracted from the original data-cubes and analyzed using the special COBE analysis software UIDL $^{1}$ written in IDL.

\subsubsection{FIR and submillimeter spectra}

FIRAS is a polarizing Michelson interferometer (Mather 1982) with two separate spectral channels (the high-frequency

\footnotetext{
${ }^{1}$ http://lambda.gsfc.nasa.gov/product/cobe/cgis.cfm
}

channel, extending from 120 to $500 \mu \mathrm{m}$ and the low-frequency channel extending from 500 to $10000 \mu \mathrm{m}$ ). The in-orbit absolute calibration of FIRAS was accomplished by using an external black-body calibrator. The spectra, already corrected for Cosmic Microwave Background and zodiac light contribution, were extracted from the original data-cubes and averaged for eight different directions on the sky over a range of 20 degrees in Galactic latitude (from -10 to 10 degrees) and from 0 to 10,5 to 15,15 to 25,25 to 35,55 to 65,85 to 95,115 to 125 , and 135 to 145 degrees in Galactic longitude.

\subsection{The $\mathrm{HI}$ and $\mathrm{H}_{2}$ maps}

The Leiden/Argentine/Bonn survey of the Galactic HI (Kalberla et al. 2005) is a merging of the Argentino de Radioastronomía and the Leiden/Dwingeloo surveys. The angular resolution of the combined survey is 0.6 square degrees (comparable to that of the COBE maps) and is the most sensitive, to date, HI map of the Milky Way.

A composite CO survey of the Milky Way constructed from 37 individual surveys has been carried out by Dame et al. $(2001)^{2}$. The total area covered by this survey is 9353 square degrees, which accounts for nearly one half of the area within 30 degrees of the Galactic plane. Using a CO-to- $\mathrm{H}_{2}$ mass conversion factor of $X=1.8 \pm 0.3 \times 10^{20} \mathrm{~cm}^{-2} \mathrm{~K}^{-1} \mathrm{~km}^{-1} \mathrm{~s}$ (Dame et al. 2001), a $\mathrm{H}_{2}$ map is constructed.

\section{The model}

The model that we use quite accurately simulates the physical processes (see Kylafis \& Bahcall 1987; and Popescu et al. 2000) that take place inside a galaxy for the wavelength range between optical and submillimeter (submm). In the optical/NIR wavelength range, absorption and scattering of the stellar light by the dust grains occurs, while emission from the heated dust grains is taking place in the FIR/submm wavelengths. The different nature of the processes allows us to treat the two wavelength ranges separately as we describe below. Since the dust reveals itself by its FIR/submm emission, it is reasonable to begin with the modeling in these wavelengths to constrain the dust parameters. By fixing the dust parameters in the radiative transfer model, we can then model the optical/NIR emission of the Galaxy.

For computational reasons, all the radiative transfer calculations are made inside a cylinder of a radius of three times the scalelength of the dust and a half-height of six times the scaleheight of the stars.

\subsection{The FIR/submm wavelength range}

The emission model that is used for the FIR/submm wavelengths is along the lines of the model described in Popescu et al. (2000; see also Misiriotis et al. 2001). Following these studies, we assume that the Galactic dust can be described by two components. The first component is the warm dust associated with star formation complexes, which is heated locally by the young stars. The second component is the cold diffuse dust, which is heated by the diffuse radiation field in the Galaxy. For the dust grain emissivity in these wavelengths, we use the values reported in Weingartner \& Draine (2001).

\footnotetext{
2 http://cfa-www.harvard.edu/mmw/ MilkyWayinMolClouds.html
} 


\subsubsection{The warm dust component}

For the density of the warm dust component we assume that its three-dimensional distribution is given by the following equation

$\rho_{w}(R, z)=\rho_{w}(0,0) \exp \left(-\frac{R}{h_{w}}-\frac{|z|}{z_{w}}\right)$,

where $R$ and $z$ are the cylindrical coordinates, $\rho_{w}(0,0)$ is the warm dust density at the center of the Galaxy and $h_{w}$ and $z_{w}$ are the exponential scalelength and scaleheight respectively. The emission of the dust depends on the temperature $T_{w}$ and on the emission cross-section per unit mass of dust $\kappa_{\lambda}$. In the wavelengths longwards of $60 \mu \mathrm{m}$, the extinction and the emission cross-sections per unit mass have the same numerical value because the albedo is negligible. Therefore, throughout the paper we will use the same symbol $\kappa_{\lambda}$ to denote the emission and extinction cross-sections of the dust. For the temperature of the warm dust $T_{w}$, we assume that it is constant throughout the Galaxy and equal to $35 \mathrm{~K}$. This is a reasonable assumption since the warm dust is mostly associated with regions of intense star formation. The spectral energy distribution (SED) of a typical star-forming region can be approximated by thermal emission from dust with a temperature of $35 \mathrm{~K}$ (see Popescu et al. 2000; and Misiriotis et al. 2004). For $\kappa_{\lambda}$ we use the $R_{V}=3.1$ model from Weingartner \& Draine (2001). Thus, at a given wavelength $\lambda$, at a given point $(R, z)$ in the Galaxy, the emission per unit volume per unit wavelength per steradian $\eta_{w}(\lambda, R, z)$ can be calculated by

$\eta_{w}(\lambda, R, z)=\rho_{w}(R, z) \kappa_{\lambda} B\left(\lambda, T_{w}\right)$,

where $B(\lambda, T)$ is the Planck function.

\subsubsection{The cold dust component}

The density of the cold dust component is described by a similar exponential disk according to the following equation

$\rho_{\mathrm{c}}(R, z)=\rho_{\mathrm{c}}(0,0) \exp \left(-\frac{R}{h_{\mathrm{c}}}-\frac{|z|}{z_{\mathrm{c}}}\right)$.

Here $\rho_{\mathrm{c}}(0,0)$ is the cold dust density at the center of the Galaxy and $h_{\mathrm{c}}$ and $z_{\mathrm{c}}$ are the exponential scalelength and scaleheight, respectively. For the temperature of the cold dust, we assume that it follows an exponential distribution within the Galactic disk, namely,

$T_{\mathrm{c}}(R, z)=\left[T_{\mathrm{c}}(0,0)-T_{\infty}\right] \exp \left(-\frac{R}{h_{T}}-\frac{|z|}{z_{T}}\right)+T_{\infty}$.

Here $h_{T}$ and $z_{T}$ are the exponential scalelength and scaleheight for the temperature of the cold dust with $T_{\mathrm{c}}(0,0)$ being the temperature at the center of the Galaxy and $T_{\infty}$ the temperature of the dust at infinity (in our case taken equal to $3 \mathrm{~K}$ ). The emission of the cold dust per unit volume per unit wavelength per steradian $\eta_{w}(\lambda, R, z)$ is given by the formula

$\eta_{\mathrm{c}}(\lambda, R, z)=\rho_{\mathrm{c}}(R, z) \kappa_{\lambda} B\left(\lambda, T_{\mathrm{c}}(R, z)\right)$,

which is identical to Eq. (2) for the warm dust component with the dust temperature now varying within the galaxy according to Eq. (4).

Given Eqs. (2) and (5), the FIR/submm intensity along any line of sight $I(l, b, \lambda)$ can be calculated by

$I(l, b, \lambda)=\int_{s_{0}}^{\text {Earth }} \mathrm{d} s\left[\eta_{w}(\lambda, R, z)+\eta_{\mathrm{c}}(\lambda, R, z)\right]$, where $l$ and $b$ are the galactic longitude and latitude, respectively. The Earth is assumed to lie exactly on the galactic plane and at a distance equal to $8 \mathrm{kpc}$ from the galactic center (Bahcall \& Soneira 1980). The lower limit of the integral $s_{0}$ is a point where the line of sight intersects a "box" inside which we assume that all the galaxy is contained.

In total, the dust spatial distribution is described by 10 parameters (namely, $\rho_{w}(0,0), h_{w}, z_{w}, \rho_{\mathrm{c}}(0,0), h_{\mathrm{c}}, z_{\mathrm{c}}, T_{\mathrm{c}}(0,0), h_{T}, z_{T}$, and $T_{w}$ ), which can be constrained using the COBE/DIRBE data at $60,100,140$, and $240 \mu \mathrm{m}$.

\subsection{The optical/NIR wavelength range}

Given the dust distribution, as this is inferred by the FIR emission, one can calculate the extinction along any line of sight at any distance from the Earth. Using this information, we calculate the observed starlight intensity along any line of sight at 1.2 and $2.2 \mu \mathrm{m}$. In particular, the intensity is given by the following equation (Kylafis \& Bahcall 1987)

$I(l, b, \lambda)=\int_{s_{0}}^{\text {Earth }} \mathrm{d} s \eta_{\mathrm{s}}(\lambda, R, z) \mathrm{e}^{-\int_{s}^{\text {Earth }} \mathrm{d} s^{\prime} \kappa_{\lambda} \rho_{\mathrm{c}}(R, z)}$.

Here $\eta_{\mathrm{s}}(\lambda, R, z)$ is the stellar emissivity at wavelength $\lambda$ at position $(R, z)$ in the galaxy, $\kappa_{\lambda}$ is the extinction cross-section per unit mass at wavelength $\lambda$ (taken from Weingartner $\&$ Draine $2001)$, and $\rho(R, z)$ is the dust density. To simplify the calculations, we assume that only the diffuse cold dust component contributes to the opacity, while the warm dust component, being associated mostly with the star-forming complexes (see Sect. 3), affects the opacity locally without affecting the global properties of the Galaxy. As we will also see later (Sect. 5), the mass of the warm dust component is more than 2 orders of magnitude less than that of the cold dust component, making it only a very small fraction of the ISM. For the spatial distribution of the stellar emissivity we follow Xilouris et al. $(1997,1998,1999)$ and assume that the stellar distribution is described by

$$
\begin{aligned}
\eta_{\mathrm{s}}(R, z)= & \eta_{\text {disk }} \exp \left(-\frac{R}{h_{\mathrm{s}}}-\frac{|z|}{z_{\mathrm{s}}}\right) \\
& +\eta_{\text {bulge }} \exp \left(-7.67 B^{1 / 4}\right) B^{-7 / 8}
\end{aligned}
$$

In this expression the first part describes an exponential disk and the second part describes the bulge, which in projection is the well-known $R^{1 / 4}$-law (Christensen 1990). In the first term of the expression, $\eta_{\text {disk }}$ is the stellar emissivity at the center of the disk, and $h_{\mathrm{s}}$ and $z_{\mathrm{s}}$ are the scalelength and scaleheight, respectively, of the stars in the disk. For the bulge, $\eta_{\text {bulge }}$ is the stellar emissivity per unit volume at the center of the bulge, while $B$ is defined by

$B=\frac{\sqrt{R^{2}+z^{2}(a / b)^{2}}}{R_{\mathrm{e}}}$,

with $R_{\mathrm{e}}$ being the effective radius of the bulge and $a$ and $b$ being the semi-major and semi-minor axis, respectively, of the bulge.

To model the Galaxy in the NIR wavelengths (and in particular at 1.2 and $2.2 \mu \mathrm{m}$ for which we have available full maps of the Galaxy), we need to calculate 9 parameters. Three of them $\left(\rho_{\mathrm{c}}(0,0), h_{\mathrm{c}}, z_{\mathrm{c}}\right.$; the parameters of the cold dust component) are constrained from the $60,100,140$, and $240 \mu \mathrm{m}$ data, as we will see later on (see Sect. 4). The remaining 6 parameters $\left(\eta_{\text {disk }}, h_{\mathrm{s}}, z_{\mathrm{s}}, \eta_{\text {bulge }}, R_{\mathrm{e}}\right.$, and $\left.a / b\right)$ are constrained from the $1.2 \mu \mathrm{m}$ map. Additional modeling is also performed on the $2.2 \mu \mathrm{m}$ map of the Galaxy (see Sect. 4). 


\subsection{The molecular and atomic hydrogen}

The two gas phases ( $\mathrm{HI}$ and $\mathrm{H}_{2}$ ), being totally independent from the dust and stars, are treated separately from the rest of the components. The distribution of the molecular hydrogen $\left(\mathrm{H}_{2}\right)$ is assumed to follow the $\mathrm{CO}$ emission. Although it is rather clumpy, it can be, to a first approximation, described by an axisymmetric exponential disk (e.g., Regan et al. 2001, for the the large-scale distribution of $\mathrm{CO}$ in other nearby galaxies). Thus, the distribution of $\mathrm{H}_{2}$ as a function of the position in the galaxy is given by

$\rho_{\mathrm{H}_{2}}(R, z)=\rho_{\mathrm{H}_{2}}(0,0) \exp \left(-\frac{R}{h_{\mathrm{H}_{2}}}-\frac{|z|}{z_{\mathrm{H}_{2}}}\right)$,

where $\rho_{\mathrm{H}_{2}}(0,0)$ is the density of the $\mathrm{H}_{2}$ molecules in the center of the galaxy, $h_{\mathrm{H}_{2}}$ is the scalelength, and $z_{\mathrm{H}_{2}}$ is the scaleheight of the distribution.

For the atomic hydrogen (HI), we assume a similar distribution, but we also introduce an inner truncation radius to account for the absence of HI in the central parts of the Galaxy, as indicated by the relevant map (see Kalberla et al. 2005). The distribution of $\mathrm{HI}$ is then given by

$\rho_{\mathrm{HI}}(R, z)= \begin{cases}\rho_{\mathrm{HI}}(0,0) \exp \left(-\frac{R}{h_{\mathrm{HI}}}-\frac{|z|}{z_{\mathrm{HI}}}\right) & , \rho>R_{t} \\ 0 & , \rho<R_{t}\end{cases}$

with $\rho_{\mathrm{HI}}(0,0)$ as the $H I$ density at the center of the galaxy (of the untruncated disk), $R_{t}$ as the inner truncation radius, $h_{\mathrm{HI}}$ as the scalelength, and $z_{\mathrm{HI}}$ as the scaleheight of the distribution. Although more sophisticated functional expressions have been suggested for the description of the HI distribution (i.e., van den Bosch 2000), we adopt the simplest possible description.

The model column density of the molecular and the atomic hydrogen along any line of sight is then calculated by

$I_{\mathrm{gas}}(l, b)=\int_{s_{0}}^{\text {Earth }} \mathrm{d} s \rho_{\mathrm{gas}}(R, z)$

with $\rho_{\text {gas }}(R, z)$ being equal to either the molecular $\rho_{\mathrm{H}_{2}}(R, z)$ or the atomic $\rho_{\mathrm{HI}}(R, z)$ hydrogen distribution (see Eqs. (10) and (11), respectively). The molecular hydrogen distribution is then parametrized by three parameters $\left(\rho_{\mathrm{H}_{2}}(0,0), h_{\mathrm{H}_{2}}\right.$, and $\left.z_{\mathrm{H}_{2}}\right)$, while the atomic hydrogen distribution, by four $\left(\rho_{\mathrm{HI}}(0,0), h_{\mathrm{HI}}\right.$, $z_{\mathrm{HI}}$, and $R_{t}$ ).

\section{The fitting procedure}

Having a computationally intensive radiative transfer model with 15 parameters in total to compute ( 9 for the dust distribution and 6 for the stellar distribution; see Sect. 3), we proceed in two steps. We first constrain the 9 parameters that define the dust distribution and the temperature of the cold dust component by fitting the model FIR intensity (Eq. (6)) to the FIR data (i.e., 60, $100,140$, and $240 \mu \mathrm{m})$. Then, having defined the dust distribution, we fit Eq. (7) to the NIR maps to constrain parameters that describe the stellar distribution.

A third step, treated separately, is to determine the parameters that describe the $\mathrm{H}_{2}$ and $\mathrm{HI}$ distributions.

In all three steps, the observed surface brightness (or the column density in the case of the gas) is compared with the computed surface brightness from the model. Before going into a detailed $\chi^{2}$ minimization to find those values of the parameters that best describe the Galaxy, we fit simple exponential profiles to the surface brightness and in directions vertical and parallel to
Table 1. Masked sources in DIRBE maps.

\begin{tabular}{lcc}
\hline \hline Source & $\begin{array}{c}\text { Central } l, b \\
\text { (degrees) }\end{array}$ & $\begin{array}{c}\text { Subtracted area } \\
\text { (sq. degrees) }\end{array}$ \\
\hline Galactic center & 0,0 & $10 \times 10$ \\
Orion arm & $-80,0$ & $20 \times 20$ \\
High latitude & 10,20 & $20 \times 20$ \\
Magellanic clouds & $80,-30$ & $20 \times 20$ \\
M31 & $-120,-20$ & $30 \times 20$ \\
\hline
\end{tabular}

Table 2. Parameters for the dust distribution (see text for a detailed description of each parameter).

\begin{tabular}{lll}
\hline \hline Parameter & Units & Value \\
\hline$\rho_{w}(0,0)$ & $\mathrm{gr} \mathrm{cm}^{-3}$ & $1.22 \times 10^{-27}$ \\
$h_{w}$ & $\mathrm{kpc}$ & 3.3 \\
$z_{w}$ & $\mathrm{kpc}$ & 0.09 \\
$\rho_{\mathrm{c}}(0,0)$ & $\mathrm{gr} \mathrm{cm}^{-3}$ & $1.51 \times 10^{-25}$ \\
$h_{\mathrm{c}}$ & $\mathrm{kpc}$ & 5.0 \\
$z_{\mathrm{c}}$ & $\mathrm{kpc}$ & 0.1 \\
$T_{\mathrm{c}}(0,0)$ & $\mathrm{K}$ & 19.2 \\
$h_{T}$ & $\mathrm{kpc}$ & 48 \\
$z_{T}$ & $\mathrm{kpc}$ & 500 \\
\hline
\end{tabular}

Table 3. Parameters for the stellar distribution (see text for a detailed description of each parameter).

\begin{tabular}{llll}
\hline \hline Parameter & Unit & Value & \\
\hline & & $(1.2 \mu \mathrm{m})$ & $(2.2 \mu \mathrm{m})$ \\
\hline$\eta_{\text {disk }}$ & $\mathrm{erg} \mathrm{s}^{-1} \mathrm{~cm}^{-3} \mathrm{~Hz}^{-1} \mathrm{srad}^{-1}$ & $5.49 \times 10^{-38}$ & $9.94 \times 10^{-38}$ \\
$h_{\mathrm{s}}$ & $\mathrm{kpc}$ & 2.5 & 2.2 \\
$z_{\mathrm{s}}$ & $\mathrm{kpc}$ & 0.16 & 0.12 \\
$\eta_{\text {bulge }}$ & $\mathrm{erg} \mathrm{s} \mathrm{cm}^{-3} \mathrm{~Hz}^{-1} \mathrm{srad}^{-1}$ & $2.07 \times 10^{-34}$ & $2.03 \times 10^{-35}$ \\
$R_{\mathrm{e}}$ & $\mathrm{kpc}$ & 0.68 & 0.79 \\
$a / b$ & - & 0.61 & 0.63 \\
\hline
\end{tabular}

the disk (see Xilouris et al. 1997, for more details). In this way, good estimates of the geometrical characteristics are derived and are used as initial guesses in a $\chi^{2}$ minimization algorithm. The minimization is done using the Steve Moshier C translation of the public domain Levenberg-Marquardt solver of the Argonne National Laboratories MINPACK mathematical library ${ }^{3}$. We always test the uniqueness of the best values of the parameters derived by the fit by altering the initial values of the fit by as much as $\sim 30-40 \%$. In all cases the values returned by the fit were the ones that we present.

For all the DIRBE maps, we used the data within the latitude range of -40 to 40 degrees. This was done because outside this latitude range the signal drops significantly with no usable input to the model. Some regions were also masked from the DIRBE maps due to their large deviation from the Galactic emission (see Table 1).

\section{Results}

Having described the way to perform the fitting of the model to the DIRBE maps (Sect. 4), we derive the values of the parameters of the model that best fit the data. The fitted parameters are presented in Tables 2-4. In Table 2 we give the values of the parameters that describe the dust distribution inside the Galactic disk. In Table 3 we present the values of the parameters for the stellar distribution as they have been determined from the 1.2

\footnotetext{
${ }^{3}$ http://www .netlib.org
} 


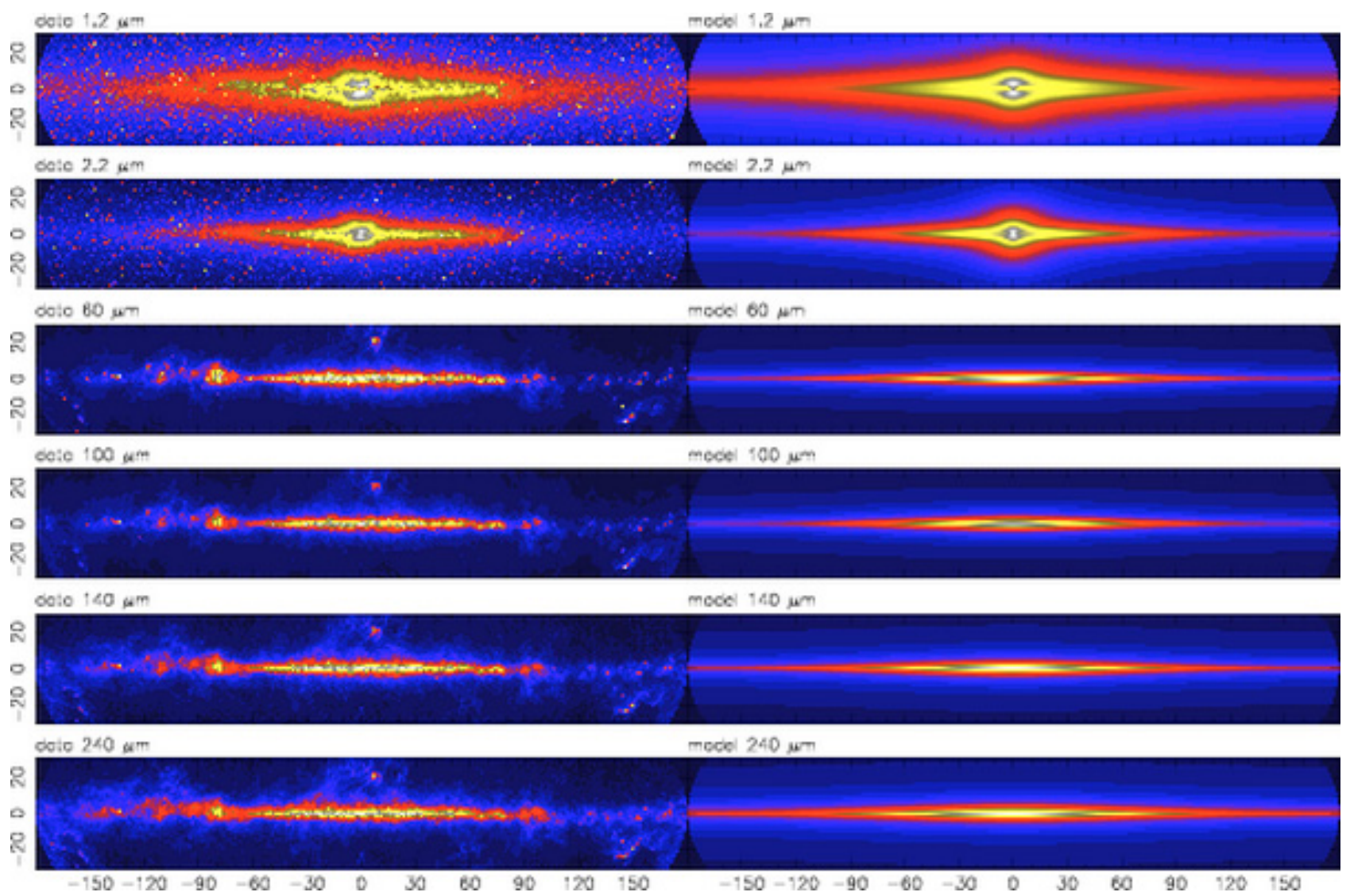

Fig. 1. The COBE/DIRBE maps of the Galaxy (left panels) in direct comparison with the fitted model (right panels). From top to bottom the 1.2, 2.2, 60, 100, 140, and $240 \mu \mathrm{m}$ maps are presented for the Galactic latitude range of -30 to 30 degrees. The maps were made in Mollweide projection (Snyder 1987).

Table 4. Parameters for gas distribution (see text for a detailed description of each parameter).

\begin{tabular}{lll}
\hline \hline Parameter & Units & Value \\
\hline$\rho_{\mathrm{H}_{2}}(0,0)$ & $\mathrm{cm}^{-3}$ & 4.06 \\
$h_{\mathrm{H}_{2}}$ & $\mathrm{kpc}$ & 2.57 \\
$z_{\mathrm{H}_{2}}$ & $\mathrm{kpc}$ & 0.08 \\
$\rho_{\mathrm{HI}}(0,0)$ & $\mathrm{cm}^{-3}$ & 0.32 \\
$h_{\mathrm{HI}}$ & $\mathrm{kpc}$ & 18.24 \\
$z_{\mathrm{HI}}$ & $\mathrm{kpc}$ & 0.52 \\
$R_{t}$ & $\mathrm{kpc}$ & 2.75 \\
\hline
\end{tabular}

and $2.2 \mu \mathrm{m}$ maps. Finally, in Table 4 we present the values of the parameters for the distribution of the molecular and the atomic hydrogen.

Having determined all the parameters for the dust and the stellar distributions, we can now create the model images of the Galaxy at each wavelength to compare with the observed images. This is what we do in Fig. 1, where the observations at different wavelengths (left panels) are compared with the model images (right panel). The 1.2, 2.2, 60, 100, 140, and $240 \mu \mathrm{m}$ images are shown from top to bottom. In these images the $x$ and $y$-axes are the Galactic longitude and latitude in degrees with the Galactic center at $(0,0)$, while positive and negative longitudes are the southern and northern regions of the sky, respectively. Positive and negative latitudes are regions above and below the Galactic plane, respectively.

As it is obvious from Fig. 1, our model and the observations compare quite well for all the different wavelengths. The
NIR $(1.2,2.2 \mu \mathrm{m})$ maps (two top panels) show the characteristic dust lane crossing the bulge along the Galactic plane which, at these wavelengths, is poorly visible (compared to the optical wavelengths). The stellar disk is well described by the smooth exponential distribution used in the model (Sect. 3). The FIR (60, 100,140 , and $240 \mu \mathrm{m}$ ) maps (third, fourth, fifth, and sixth panels from the top, respectively) show the diffuse dust emission along the Galactic plane. Given the simplicity of the axisymmetric distributions that were used, the model quite accurately reproduces the observed emission at these wavelengths.

To get a better view of the goodness of the fit of the model to the data, we produce vertical profiles of the surface brightness along 10 meridian zones of 10 degrees width each. This is shown in Fig. 2, where in each panel we overplot the model to the data for each wavelength $(1.2,2.2,60,100,140$, and $240 \mu \mathrm{m}$, as indicated with the wavelength value inside each panel). In each panel (of different wavelength) the averaged profiles along the longitude meridians of $0,5,10,20,30,60,90,120,140$, and 180 degrees are presented shifted with each other by a factor of 10 in surface brightness for reasons of clarity. As one can see, the agreement between the model and the observations is quite good.

We note here that in the first two panels of Fig. 2 the absorption by dust is seen as a dip in the first few meridian profiles. The dip is more prominent at $1.2 \mu \mathrm{m}$ than at $2.2 \mu \mathrm{m}$. The $60 \mu \mathrm{m}$ emission is mainly due to the warm dust that traces regions of star formation. The $240 \mu \mathrm{m}$ emission traces the diffuse cold dust.

To get a better feeling of the goodness of the fit, we present the relative percentage of the residuals as a function of the percentage of the Galaxy's area. We do that in Table 5 where, for 


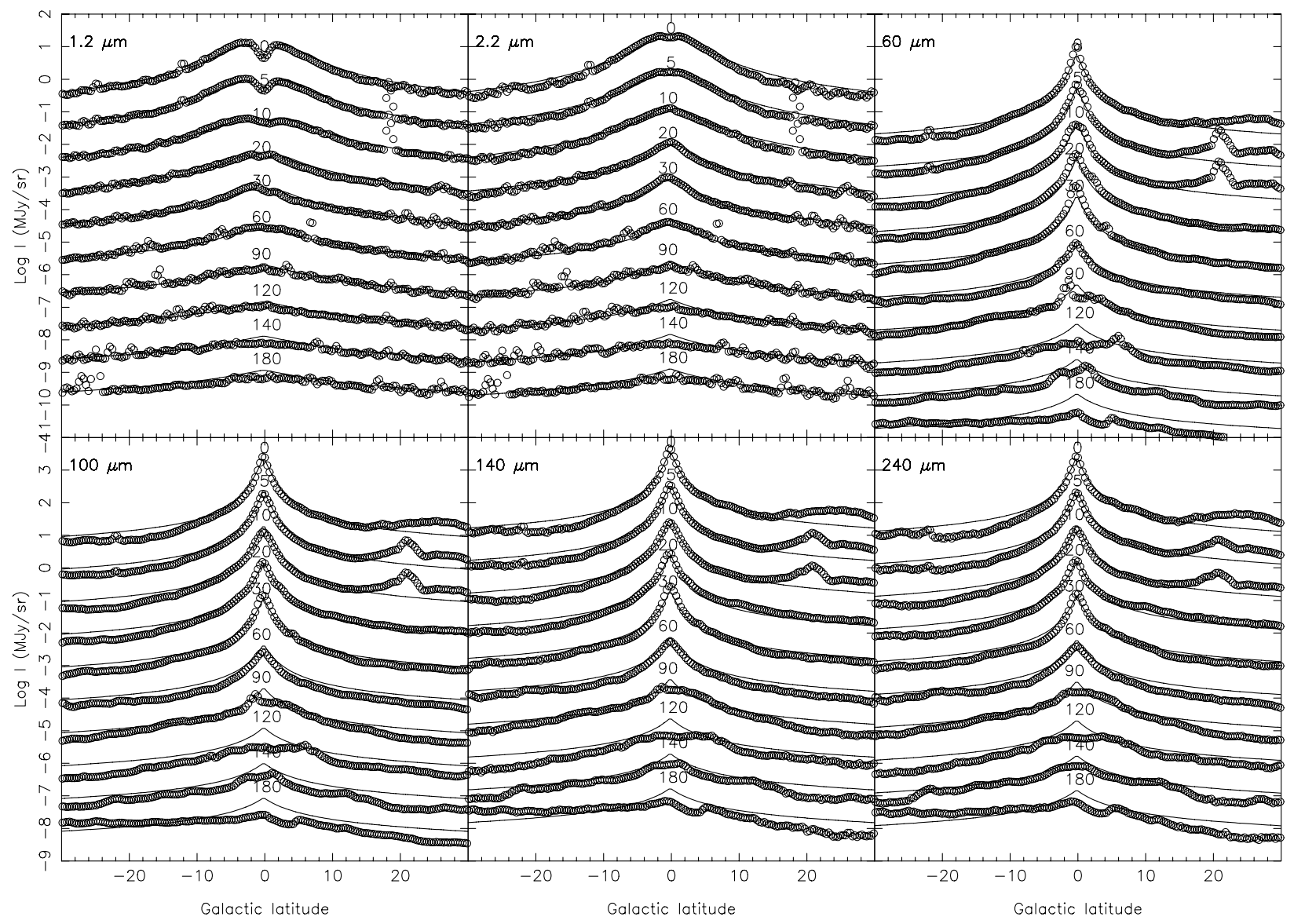

Fig. 2. Profiles along Galactic meridian zones (circles) together with the corresponding models (solid lines). Taking advantage of the symmetry of the Galaxy, we have folded the maps over the central meridian and extracted 10 meridian profiles (centered at 0, 5, 10, 20, 30, 60, 90, 120, 140, and 180 degrees in Galactic longitude). Each profile is averaged over a 10 degree zone in Galactic longitude. Due to the folding of the map, the zero longitude profile and the 180 longitude profile come from the $0-5$ and 175-180 meridian zones, respectively. From top left to bottom right we show the profiles at 1.2, 2.2, 60, 100,140, and $240 \mu \mathrm{m}$ (as indicated in the upper left corner of each panel).

Table 5. Residuals between the model and the observation. For each COBE/DIRBE map we present the percentage of the Galaxy's area with residuals less than $<10 \%,<20 \%$, and $<50 \%$.

\begin{tabular}{lccc}
\hline \hline Wavelength $(\mu \mathrm{m})$ & $<10 \%$ & $<20 \%$ & $<50 \%$ \\
\hline 1.2 & $29 \%$ & $53 \%$ & $92 \%$ \\
2.2 & $32 \%$ & $55 \%$ & $87 \%$ \\
60 & $26 \%$ & $52 \%$ & $88 \%$ \\
100 & $23 \%$ & $43 \%$ & $81 \%$ \\
140 & $28 \%$ & $51 \%$ & $81 \%$ \\
240 & $18 \%$ & $37 \%$ & $75 \%$ \\
\hline $\mathrm{HI}$ & $17 \%$ & $38 \%$ & $85 \%$ \\
$\mathrm{H}_{2}$ & $17 \%$ & $35 \%$ & $67 \%$ \\
\hline
\end{tabular}

example, for the 1.2 micron band we have $29 \%$ of the area of the Galaxy with residuals less than $10 \%, 53 \%$ of the area with residuals less than $20 \%$, and $92 \%$ of the area of the Galaxy with residuals less than $50 \%$. The same holds for the rest of the bands. We see that, on average, about $50 \%$ (half of the Galaxy's area) has residuals less than $20 \%$. Given the large noise measurements that exist in the high latitude regions (especially in the FIR maps), and also the complexity of the real structures, these numbers show how well the model fits the real data.

Using the values of the parameters presented in Table 2 and adopting the Weingartner \& Draine (2001) values for the extinction cross-section at $1.2 \mu \mathrm{m}$ and at $2.2 \mu \mathrm{m}$, we can calculate the optical depth (and subsequently the extinction) between any two points in the Galaxy. In particular, the central face-on optical depth $\tau_{\lambda}^{f}=2 \kappa_{\lambda} z_{\mathrm{c}}$ is 0.33 at $1.2 \mu \mathrm{m}$ and 0.17 at $2.2 \mu \mathrm{m}$.

In Fig. 3 we compare the maps of the hydrogen column density with the corresponding model. The top panel on the left shows the molecular hydrogen map as this is inferred from the CO observations (Dame et al. 2001). The respective model column density is shown in the top right panel. From the comparison between the model and the observations it is evident that, despite the large clumpiness of the data, the large-scale structure is fairly well represented by the model. In the bottom left panel we show the map of the atomic hydrogen (Kalberla et al. 2005). The lack of this component in the central part of the galaxy shows up as a decrease of the column density. The model column density is shown in the bottom right panel. In this image, the lack of atomic hydrogen in the center of the Galaxy is more prominent due to the sharp cut introduced by the model at the truncation radius $R_{t}$.

The comparison between the model and the data is shown better in Fig. 4. The left panel shows vertical profiles of the column density of the atomic hydrogen along 10 meridian zones of width 10 degrees each. The agreement between the model and the data is good. The observed CO (and subsequently the $\mathrm{H}_{2}$ ) distribution on the other hand is very clumpy as it can be seen from the noise of the data in the profiles shown in the right 


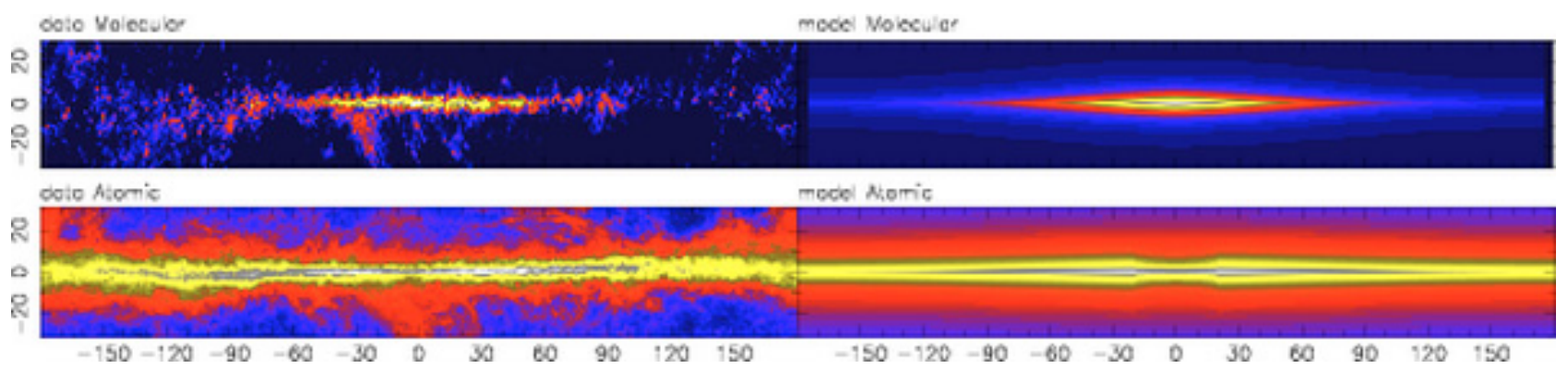

Fig. 3. The gas maps of the Galaxy (left panels) in direct comparison with the fitted model (right panels). The upper left panel shows the molecular hydrogen distribution (Dame et al. 2001), while the atomic hydrogen distribution (Kalberla et al. 2005) is presented in the bottom left panel. Both maps are given for the Galactic latitude range of -30 to 30 degrees.

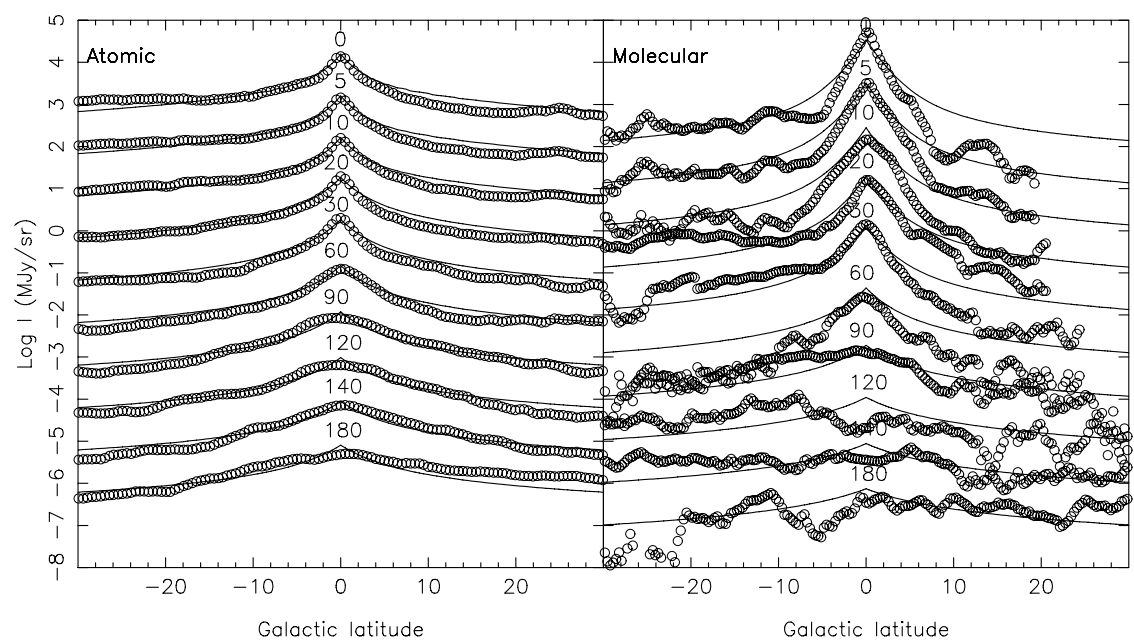

Fig. 4. Profiles along Galactic meridian zones (circles) together with the corresponding models (solid lines). As in the case of the COBE/DIRBE data (Fig. 2), the maps are folded over the central meridian and 10 meridian profiles are extracted following the same method described in Fig. 2. The left panel shows the atomic hydrogen profiles, and the right panel shows the molecular hydrogen profiles.

panel of Fig. 4. The smooth model, however, follows the actual distribution fairly well.

As in the case of the COBE/DIRBE maps, we also present the residuals (in percentage) between the model and the observations as a function of the Galaxy's area. We do that in Table 5 (last two rows).

Having determined the geometrical characteristics of the distributions and the central densities, it is then straightforward to compute the total mass of the cold dust $M_{\mathrm{c}}$, the warm dust $M_{w}$, the mass of the molecular hydrogen $M_{\mathrm{H}_{2}}$, and the mass of the atomic hydrogen $M_{\mathrm{HI}}$ by integrating the respective three dimensional distributions in space. For distributions of the form of Eqs. (1), (3), and (10) for the warm dust, the cold dust, and the molecular hydrogen, respectively, this integration gives

$M=4 \pi \rho(0,0) z h^{2}$,

with $h$ being the scalelength of the distribution, $z$ the scaleheight, and $\rho(0,0)$ the central density of the material. If we also include an inner cut-off in the distribution with a truncation radius $R_{t}$, as we do for the atomic hydrogen (Eq. (11)), the integration then gives

$M=4 \pi \rho(0,0) z h\left(R_{t}+h\right) \exp \left(-\frac{R_{t}}{h}\right)$.

Using the values for the fitted parameters presented in Tables 2 and 4 and the above equations, we then find $M_{\mathrm{c}}=7.0 \times$ $10^{7} M_{\odot}, M_{w}=2.2 \times 10^{5} M_{\odot}, M_{\mathrm{H}_{2}}=1.3 \times 10^{9} M_{\odot}$, and $M_{\mathrm{HI}}=8.2 \times 10^{9} M_{\odot}$. The total gas-to-dust mass ratio becomes $\left(M_{\mathrm{H}_{2}}+M_{\mathrm{HI}}\right) /\left(M_{\mathrm{c}}+M_{w}\right)=135$, which is in fair agreement with the value of $160 \pm 60$ reported in Sodroski et al. (1994).

\section{Discussion}

\subsection{Comparison of the model with the FIRAS data}

To further establish the validity of the model, we compare it with the COBE/FIRAS data. In Fig. 5 we present the spectrum of the Galaxy in several directions along the Galactic plane averaged over an area of \pm 10 degrees in latitude and 10 degrees in longitude centered on the Galactic equator and at 5, 10, 20, 30, 60, 90,120 , and 140 degrees in longitude. On average, the model is in good agreement with the data. The deviation in some cases is to be expected due to the non-axisymmetric nature of the real distribution of the dust.

Evidence supporting the previous statement comes from a comparison of the "global" SED of the Milky Way constructed by averaging the COBE/FIRAS maps in all the directions on the sky with the predicted SED from our model. This is shown in Fig. 6 (top panel).

The goodness of the fit can be evaluated by looking at the residuals between the model and the observations which are presented in the bottom panel of Fig. 6. From this plot we see that $82 \%$ of the data points on this SED show residuals from the modeled SED that are less than $10 \%$. In particular, $14 \%$, $24 \%, 47 \%, 82 \%$, and $93 \%$ of the data points show residuals 


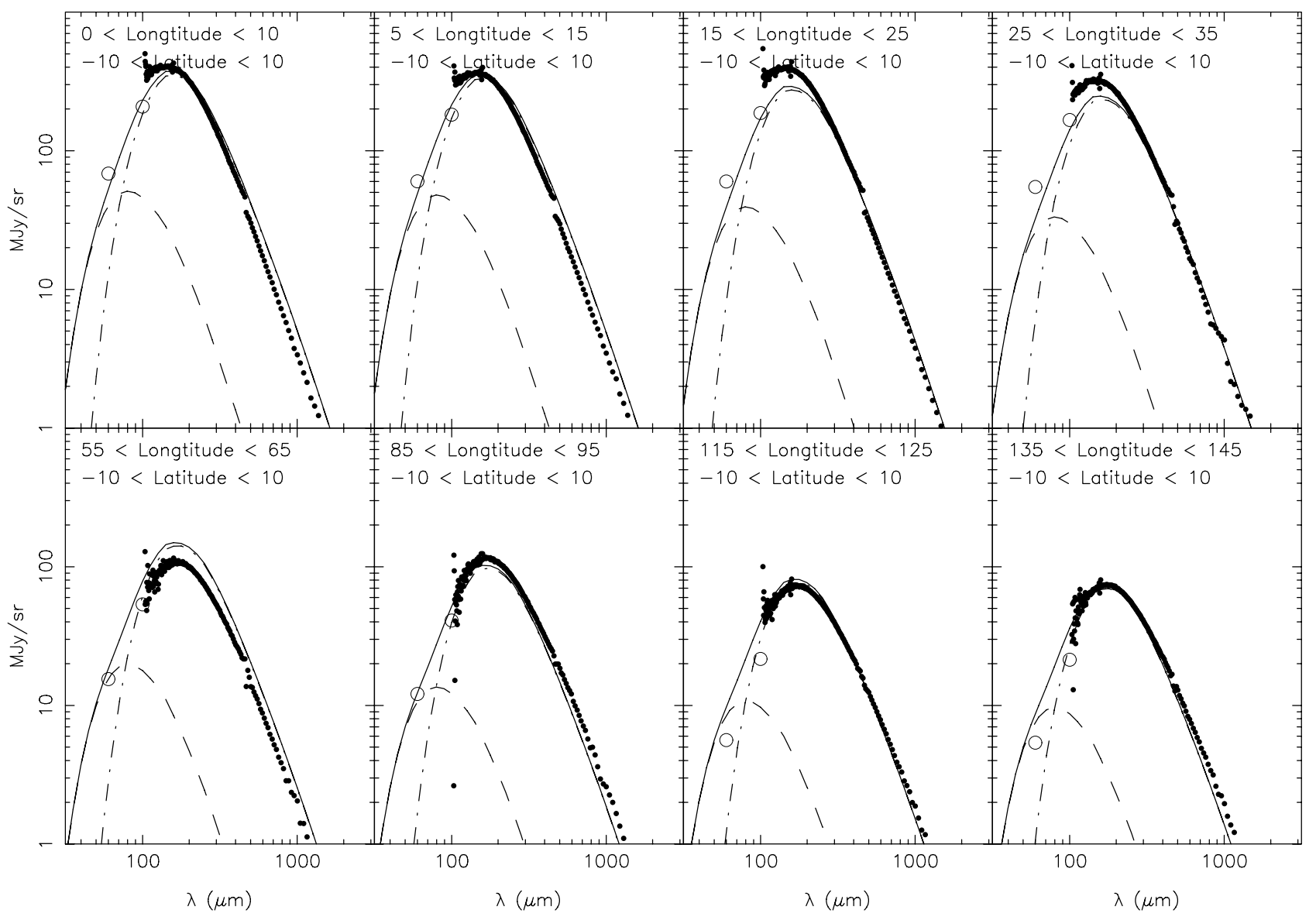

Fig. 5. The Spectral Energy Distribution (SED) of the Milky Way averaged between -10 and 10 degrees in Galactic latitude and over 10 degrees in Galactic longitude, as indicated on top of each panel. In each panel the filled circles are the COBE/FIRAS data and the open circles are the COBE/DIRBE data. The dashed line is the contribution to the SED of the warm dust component, the dot-dashed line is the contribution of the cold dust component and the solid line is the total modeled SED.

from the model which are less than $1 \%, 2 \%, 5 \%, 10 \%$, and $20 \%$, respectively.

This agreement, which does not come from fitting the model to the data, but from comparison of the data with our model, gives us confidence that our model gives a consistent representation of the Galaxy in wavelengths extending up to $1000 \mu \mathrm{m}$.

In their analysis, Reach et al. (1995) reported emission from a very cold component $(4-7 \mathrm{~K})$. This component is not evident in our analysis when looking at the averaged SED of the Galaxy (Fig. 6). However, deviations though between the model and the observations do exist when looking through various lines-ofsight (Fig. 5), which can, as said earlier, be explained by local deviations of the density of the dust material.

\subsection{Temperature distribution}

As already mentioned in Sect. 3.1, the dust in our model is described by two components (one with a constant "warm" temperature of $35 \mathrm{~K}$, associated with the warm HII star-forming regions, and another "cold" temperature component). For the distribution of the temperature of the cold dust material, we assume a simple exponential distribution in both directions, radially and vertically to the plane of the Galactic disk (see Eq. (4)), with a boundary condition that the temperature at large distances is at $3 \mathrm{~K}$ (of course, as noted earlier, the radiative transfer calculations only take place within a finite volume where dust material exists; see Sect. 3). To better visualize the spatial distribution of the temperature of the cold dust component we plot the temperature map seen in Fig. 7. This map represents a two dimensional slice of the Galactic plane that extends up to $20 \mathrm{kpc}$ along and up to $0.3 \mathrm{kpc}$ above the Galactic plane with the temperature contours plotted every $0.5 \mathrm{~K}$. The radial profiles of the cold dust density (in units of $5 \times 10^{-25} \mathrm{gr} / \mathrm{cm}^{3}$ ) are also overplotted for $z=0.0$, $z=0.1 \mathrm{kpc}$, and $z=0.2 \mathrm{kpc}$ indicating the way that the dust material is distributed within this temperature field. From this plot we see that the temperature varies from $19.2 \mathrm{~K}$ at the center to $\sim 15 \mathrm{~K}$ at the outer parts of the Galaxy $(\sim 15 \mathrm{kpc})$. This result agrees with the studies of Reach et al. (1995), Sodrosky et al. (1994), and Davies et al. (1997) showing the similar radial dependence of the cold dust component. Concerning the vertical dependence of the temperature of the cold component, we see that this is more or less constant for different heights above the Galactic plane. We note here though that for higher opacities this picture could be different, with the dust being more effectively heated in places above the disk compared with the dust in the Galactic plane, where the dust is shielded from the diffuse radiation field (see Bianchi et al. 2000).

\subsection{Star formation efficiency in the Milky Way disk and the Schmidt law}

As already mentioned in the Introduction, a good indicator of the SFR is the FIR emission (e.g., Hunter et al. 1986; Lehnert \& Heckman 1996; Meurer et al. 1997; Kennicuut 1998b; 

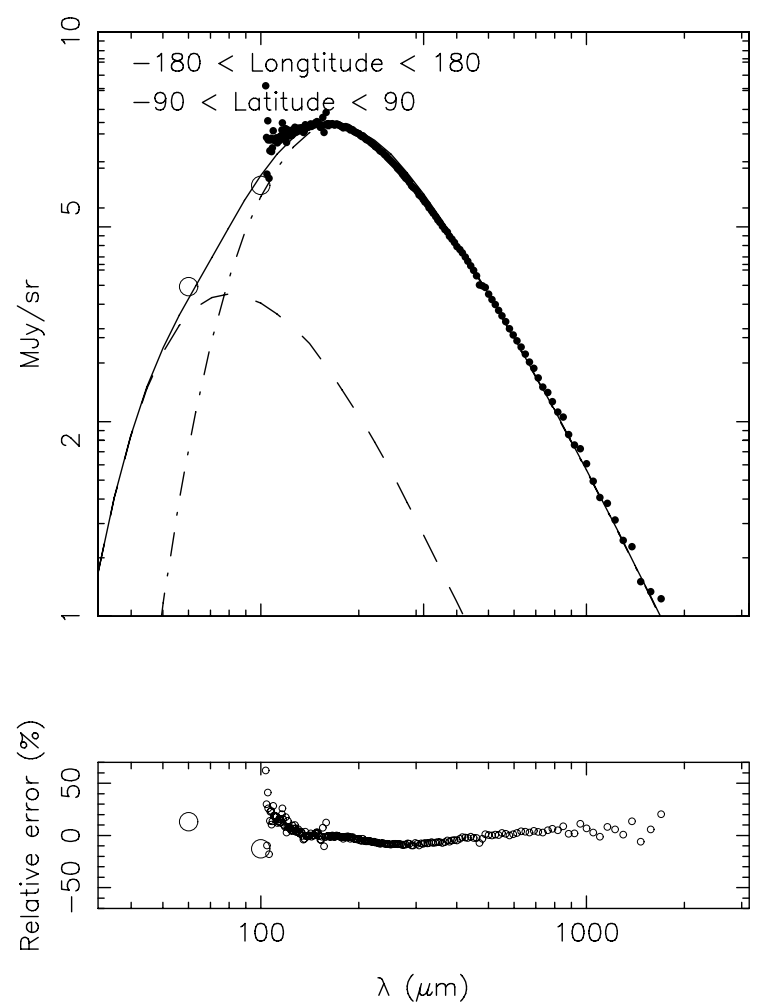

Fig. 6. The global SED of the Milky Way averaged over the whole sky (top panel). As in Fig. 5, the filled circles are the COBE/FIRAS data and the open circles are the COBE/DIRBE data, while the dashed line is the contribution to the SED of the warm dust component, the dot-dashed line is the contribution of the cold dust component, and the solid line is the total modeled SED. In the bottom panel we present the residuals (in percentage) between model and real measurements.

Kewley et al. 2002). To exploit the FIR emission we make use of the strong correlation between the SFR and the $100 \mu \mathrm{m}$ flux found in Misiriotis et al. (2004) for a large sample of spiral galaxies. Using this relation and calculating the total $100 \mu \mathrm{m}$ luminosity, we derive a SFR of $2.7 M_{\odot} \mathrm{yr}^{-1}$ for the Milky Way.

The SFR surface density can then be calculated in the disk of the Milky Way, using the same relation presented in Misiriotis et al. (2004), and plotted as a function of the Galactic radius. This is done in Fig. 8, where our model prediction (solid line) is compared with several estimates of the SFR found in the literature (see Boissier \& Pratzos 1999, and references therein). Both the SFR normalized to the value at the galactocentric distance of $8 \mathrm{kpc}\left(\mathrm{SFR}_{\odot}\right)$ and the star formation rate density are presented in this plot as a function of $\mathrm{R}$, with the model showing an excellent agreement with the existing estimates of the various SFR tracers to at least two kpc from the center. The inner region shows, acknowledging the large uncertainty of the few data points available, a decrease of the SFR efficiency, presumably due to a non-exponential distribution of the dust in such small scales. Lack of dust, for example, in this region may be due to the presence of the bar structure of the Galaxy. As mentioned earlier though, the purpose of this study is to keep the modeling as simple as possible with the aim of deriving a valuable description of the Galaxy. With this goal in mind we avoid going into a more sophisticated modeling of the central part.

A simple power-law relation between SFR and the gas content of external galaxies, introduced by Schmidt (1959) and further explored by Kennicutt (1998a), is well established and tested for large samples of galaxies (Misiriotis et al. 2004). This relation is expressed in terms of the SFR surface density $\Sigma_{\text {SFR }}$ and the gas surface density $\Sigma_{\text {gas }}$ and has the form:

$\Sigma_{\mathrm{SFR}}=A \Sigma_{\text {gas }}^{N}$,

with $A$ being the absolute SFR efficiency and $N$ the slope of the power-law. Kennicutt (1998a) examined two samples of galaxies showing normal and starbursting behavior and derived a Schmidt law for normal galaxies (with $N=2.47 \pm 0.39$ ) and for starburst galaxies (with $N=1.40 \pm 0.13$ ).

In our study we have, for the first time, the opportunity to examine the validity of this law not for a sample of external galaxies, by measuring their global properties, but for different regions of the same galaxy (the Milky Way). This is shown in Fig. 9 with the SFR surface density $\left(\Sigma_{\text {SFR }}\right)$ calculated for different radii along the Galactic disk, plotted against the gas surface density $\Sigma_{\text {gas }}$ of the same region. These data are shown with the numbers close to the points indicating the distance to the Galactic center (in kpc). Furthermore, it is evident that for regions within the Galaxy with small gas content (at the outer part of the disk) the SFR is low, while it gets more intense for regions with larger gas density (close to the center of the galaxy). From this plot it is evident that the Galactic Schmidt law also follows a power law. A best fit to these data (indicated with a solid line in the plot) yields

$$
\frac{\Sigma_{\mathrm{SFR}}}{M_{\odot} \mathrm{yr}^{-1} \mathrm{pc}^{-2}}=4.45 \times 10^{-5}\left(\frac{\Sigma_{\mathrm{gas}}}{M_{\odot} \mathrm{kpc}^{-2}}\right)^{2.18} \text {. }
$$

The power-law index of $2.18 \pm 0.20$ that we find is in a very good agreement with the value of $2.47 \pm 0.39$ quoted by Kennicutt (1998a) for normal galaxies. This is also evident by looking at the distribution of the normal galaxies on this plot (Fig. 9). The points for the external galaxies are well correlated with the measurements for different regions within the Milky Way. This indicates the strong correlation between the gas content and the SFR activity. The striking similarity of the "internal" Schmidt law for an individual galaxy (in this case the Milky Way) and the "external" Schmidt law for the global properties of normal galaxies may indicate a universal gas-SFR correlation not only on a large scale (for galaxies as a whole), but also on a small scale (for different regions inside a galaxy).

Another very useful parameter that describes the current starforming activity of a galaxy is the star formation efficiency $(\epsilon)$. In Fig. 9 the three parallel, lines correspond to constant SFRs per unit gas mass in units of $1 \%, 10 \%$, and $100 \%$ per $0.1 \mathrm{Gyr}$, as described in Kennicutt (1998a). It is interesting to notice that the star formation efficiency of the Milky Way goes from $\sim 1 \%$ per $0.1 \mathrm{Gyr}$ in the outer regions $(\sim 14 \mathrm{kpc})$ to $\sim 10 \%$ per $0.1 \mathrm{Gyr}$ in the center. This means that the Galaxy converts $\sim 1 \%$ of the gas in the outer parts of the disk $(\sim 10 \%$ in the center) to stars over the period of $0.1 \mathrm{Gyr}$, which roughly corresponds to one orbital period of the disk.

The average global efficiency of star formation in the Milky Way can be calculated by dividing the SFR over the period of 0.1 Gyr $\left(2.7 \times 10^{8} M_{\odot}\right.$; see beginning of Sect. 6.3) by the total gas mass $\left(M_{\mathrm{H}_{2}}+M_{\mathrm{HI}}=9.5 \times 10^{9}\right)$. This results in $2.8 \%$ per $0.1 \mathrm{Gyr}$ or, in other words, that our Galaxy spends $2.8 \%$ of its gas to create stars over $0.1 \mathrm{Gyr}$. This is at the lower end of the median star formation efficiency of typical present-day spiral galaxies, which is $4.8 \%$ (Kennicutt 1998a).

\subsection{Comparing the model with optical data}

To check the validity of our model in the optical wavelengths, we use the Database of Galactic Classical Cepheids 


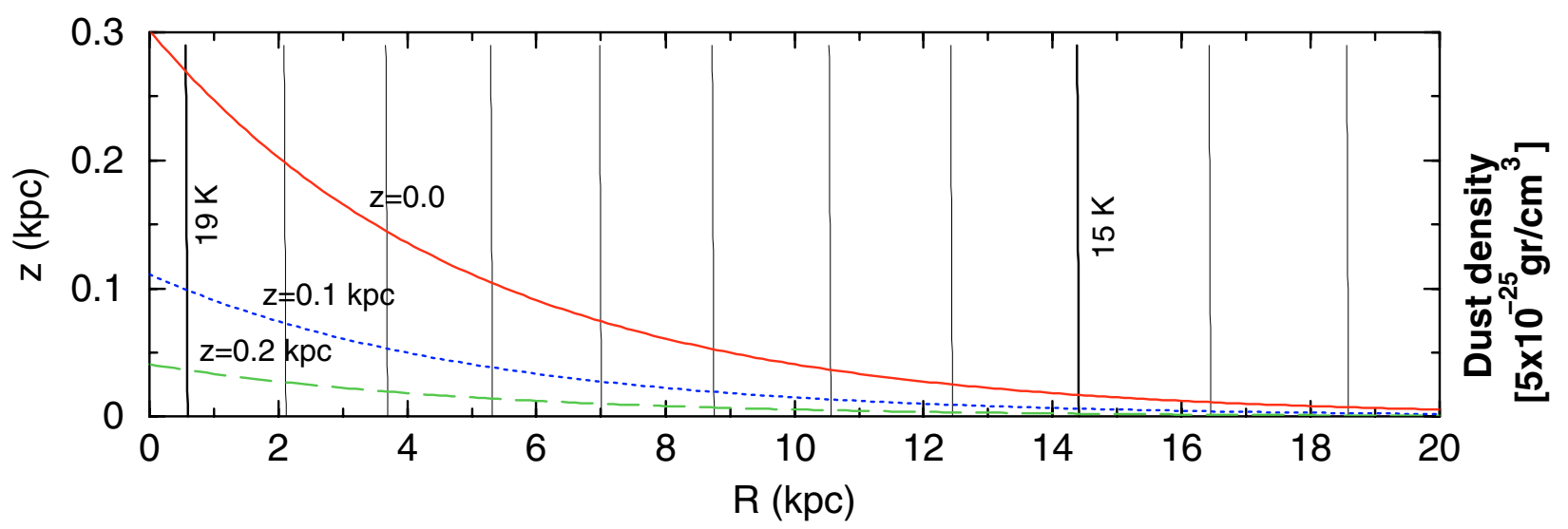

Fig. 7. Temperature map of the cold dust component within the Milky Way. Temperature contours (the almost vertical black solid lines) are plotted every $0.5 \mathrm{~K}$ and highlighted at $15 \mathrm{~K}$ and $19 \mathrm{~K}$ by a label and a thicker line. The radial profiles of the cold dust density are also overplotted for $z=0.0$ (red solid line), $z=0.1 \mathrm{kpc}$ (blue dotted line), and $z=0.2 \mathrm{kpc}$ (green dashed line) in units of $5 \times 10^{-25} \mathrm{gr} / \mathrm{cm}^{3}$.

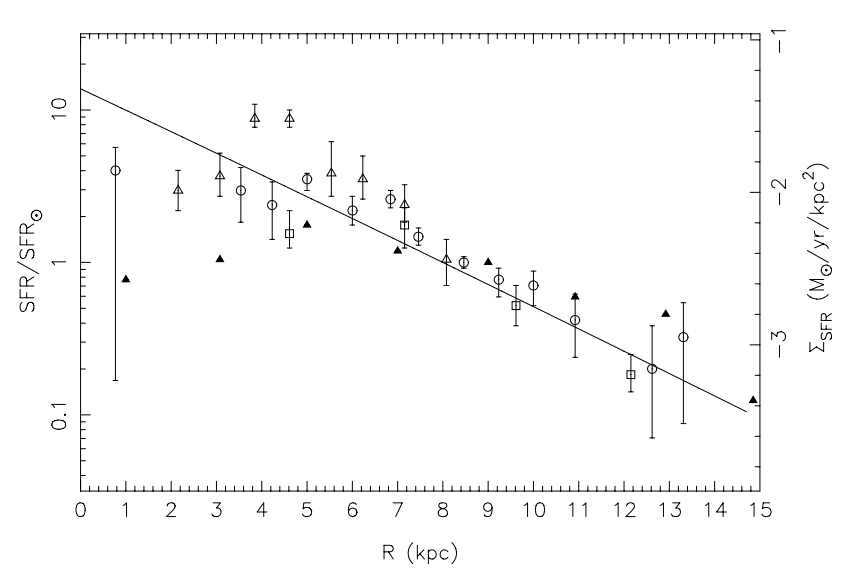

Fig. 8. The star formation rate (SFR) normalized to the value at the galactocentric distance of $8 \mathrm{kpc}\left(\mathrm{SFR}_{\odot} ; y\right.$-axis on the left) and the star formation rate density $\left(\Sigma_{\mathrm{SFR}} ; y\right.$-axis on the right) as a function of radius in the Milky Way. The data correspond to different star formation estimates and are taken from Lyne et al. (1985, open circles), Guesten \& Mezger (1982, triangles), and Guibert et al. (1978, squares; see Fig. 2 of Boissier \& Prantzos 1999). The solid line corresponds to the model prediction.

(Fernie et el. 1995). This database contains information about over 500 classical Cepheids in the Galaxy with determined distances ranging from a couple of hundred parsec up to $\sim 6.5 \mathrm{kpc}$. The $E(B-V)$ color excess reported in this catalog for each star was then checked against the $E(B-V)$ determined with our model by calculating for each Cepheid the extinction in the $B$ - and $V$-bands. The $B$ and $V$ extinction maps for the Galaxy were created by assuming the values of $h_{\mathrm{c}}$ and $z_{\mathrm{c}}$ given in Table 2 and using the values of the extinction coefficient for the $B$ and $V$-bands calculated in Weingartner \& Draine (2001). The comparison between the observed data and the model predictions for $E(B-V)$ is shown in Fig. 10 with the $x$-axis being the $B-V$ color excess observed and the $y$-axis the $B-V$ color excess predicted by the model. From this plot it is evident that the correlation between the observed and the predicted measurements is extremely good with the average of the points, binned every $0.2 \mathrm{mag}$ of $E(B-V)$ on both axes, falling, within the error bars, along the diagonal of the plot. This is also evident by looking at the individual observations that are scattered symmetrically with respect to the diagonal.

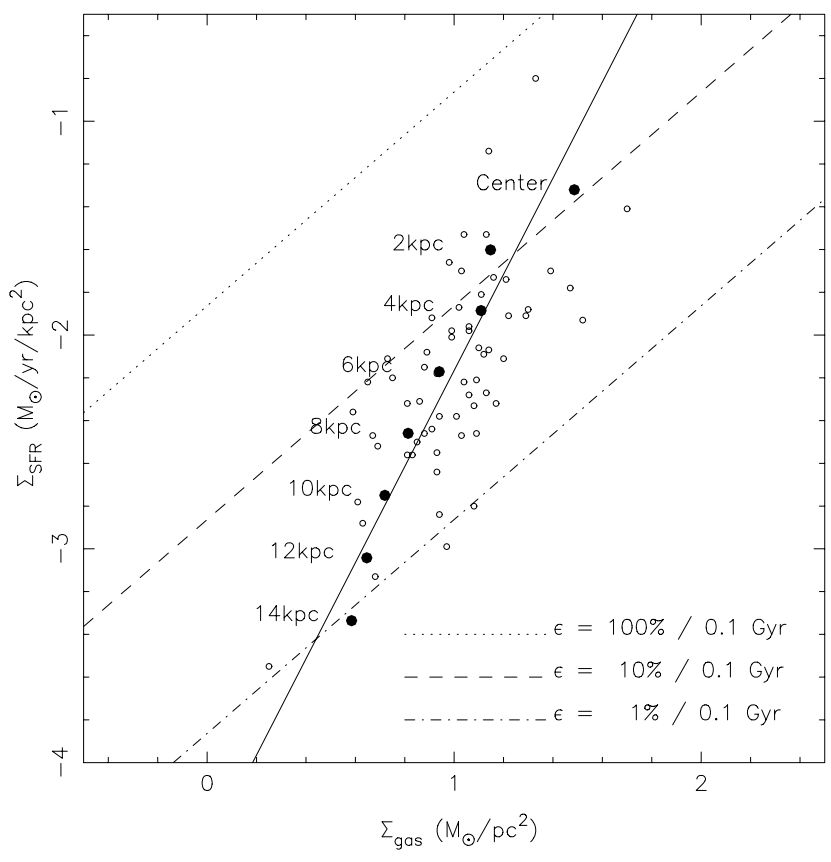

Fig. 9. Star formation rate density as a function of gas surface density in the Milky Way disk. The solid circles indicate the measurements for the Milky Way (for distances along the disk as indicated by the numbers close to the solid circles), while the solid line is the best fit of a Schmidt law to these points (see the text for details). The open circles are the global measurements for a sample of external galaxies presented in Kennicutt (1998a). The three parallel dashed and dotted lines correspond to constant star formation efficiencies of $1 \%, 10 \%$, and $100 \%$ per $0.1 \mathrm{Gyr}$.

The scatter of the points indicates that in many cases our model either overestimates or underestimates the amount of dust along the line of sight. This is clear evidence of the clumpy distribution of the dust. However, the fact that the opacity overestimated lines of sight are about as many as the underestimated ones ensures that on average our smooth model is consistent with the observed extinctions.

\section{Conclusions}

In the present study a consistent model of the ISM for a wide range of the spectrum (from optical wavelengths to millimeter 


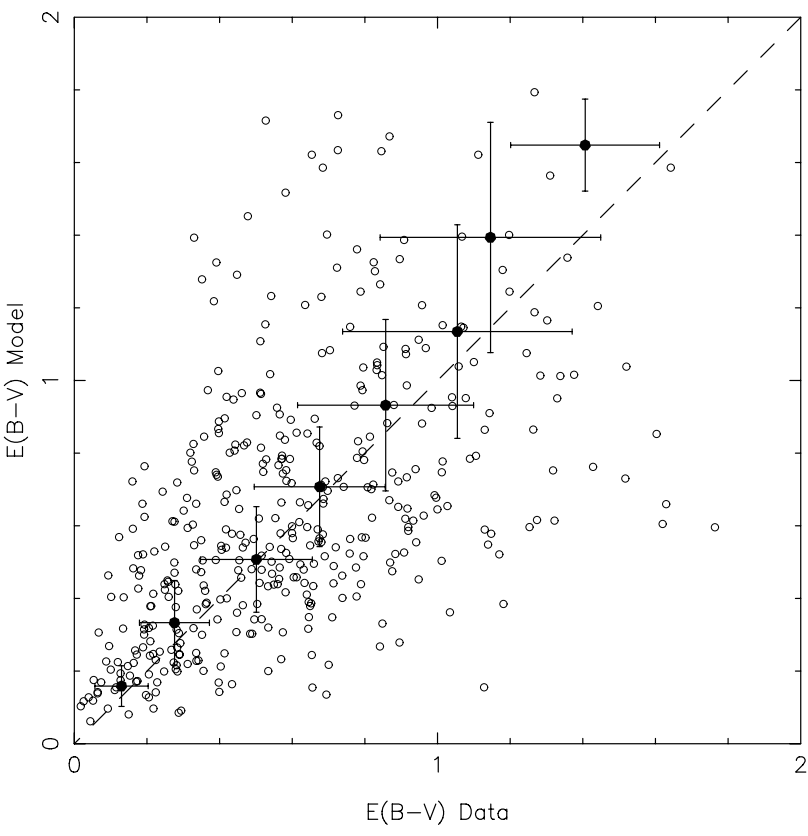

Fig. 10. The $B-V$ color excess for the sample of the Galactic Classical Cepheids (Fernie et al. 1995; $x$-axis) compared with the color excess along the same directions as derived from the model.

wavelengths) is presented. Using a simple axisymmetric threedimensional radiative transfer model for the dust and the stars in the Galactic disk, we derive the set of the parameters that best describes the FIR emission observed by COBE/DIRBE at 60 , 100,140 , and $240 \mu \mathrm{m}$. Having constrained the dust distribution, we then fit the NIR COBE/DIRBE observations at 1.2 and $2.2 \mu \mathrm{m}$ and determine the NIR properties of the Galaxy. The scalelength for the stars at 1.2 and $2.2 \mu \mathrm{m}$ is found to be 2.5 and $2.2 \mathrm{kpc}$ respectively. This comes in agreement with findings of recent studies (see Drimmel \& Spergel 2001, and references therein). Having calculated both the NIR bands, we see the trend of increasing scalelength with decreasing wavelength. This trend is also found in other sudies of nearby galaxies (e.g., De Jong 1996; Xilouris et al. 1999). We find that the ratio of the stellar to the dust scaleheight is $\sim 1.7$, while the scalelength of the dust is about twice that of the stars (as inferred from the NIR observations). Given the fact that we expect larger scalelength values in the optical (by as much as $20 \%$ ), this scalelength ratio could become $\sim 1.4-1.5$ in the optical wavelengths (see Davies et al. 1997; Xilouris et al. 1999). In addition to the COBE data, we also model the 3D distribution of the atomic and molecular hydrogen as they reveal themselves from the $21 \mathrm{~cm}$ and the $\mathrm{CO}$ emissions, respectively. We then compare our model predictions (for wavelengths up to $1000 \mu \mathrm{m}$ ) with the observations by COBE/FIRAS. The global SED of the Galaxy (averaged over the entire Galaxy) is in excellent agreement with the model, while the model shows small deviations from the SED of specific directions on the sky. These could be explained by assuming local deviations of the density of the dust (clumpiness of the dust). The star formation rate as a function of Galactocentric distance is then derived by the model and is found to be in excellent agreement with various star formation tracers within the Galaxy. Comparing the SFR surface density with the gas surface density, an "internal" Schmidt law is derived for the regions along the Galactic disk that is almost identical to the "external" Schmidt law derived by Kennicutt (1998a) for typical spiral galaxies. This could be an indication of a universal gas-SFR behavior not only on large scales (for the galaxies as a whole), but also on small scales (within an individual galaxy). The star formation efficiency of the Milky Way is found to decrease from $10 \%$ to $1 \%$ per 0.1 Gyr when going from the center to the outer parts of the Galactic disk. Finally the model predictions in the optical wavelengths ( $B$ - and $V$-bands) are compared with existing observations of Cepheid stars (in terms of their observed $E(B-V)$ color excess) and a good correlation (within the statistical errors) is found.

Acknowledgements. We thank N. D. Kylafis and S. Bianchi for their significant contribution in the final stages of this work. We would also like to thank V. Charmandaris for useful discussions concerning the FIRAS data and P. M. W. Kalberla for kindly providing us with the map of the Leiden/Argentine/Bonn Survey of Galactic HI. We acknowledge the use of the Legacy Archive for Microwave Background Data Analysis (LAMBDA). Support for LAMBDA is provided by the NASA Office of Space Science. We would like to thank the anonymous referee for pointing several weaknesses in the early version of this paper. This work has been supported in part by a Pythagoras II research program of the Ministry of Education of Greece.

\section{References}

Bahcall, J. N., \& Soneira, R. M. 1980, ApJ, 238, 17

Bianchi, S., Davies, J. I., \& Alton, P. B. 2000, A\&A, 359, 65

Boggess, N. W., Mather, J. C., Weiss, R., et al. 1992, ApJ, 397, 420

Boissier, S., \& Prantzos, N. 1999, MNRAS, 307, 857

Christensen, H. J. 1990, MNRAS, 246, 535

Dame, T. M., Hartmann, Dap., \& Thaddeus, P. 2001, ApJ, 547, 792

Davies, J. I., Trewhella, M., Jones, H., et al. 1997, MNRAS, 288, 679

De Jong, R. S. 1996, A\&A, 313, 45

Drimmel, R., \& Spergel, D. N. 2001, ApJ, 556, 181

Drimmel, R., Cabrera-Lavers, A., \& López-Corredoira, M. 2003, A\&A, 409, 205

Fernie, J. D., Beattie, B., Evans, N. R., \& Seager, S. 1995, IBVS No. 4148

Fixsen, D. J., Cheng, E. S., Gales, J. M., et al. 1996, ApJ, 473, 576

Guesden, R., \& Mezger, P. G. 1982, Vistas Astron., 26, 159

Guibert, J., Lequeux, J., \& Viallefond, F. 1978, A\&A, 68, 1

Hunter, D. A., Gillett, F. C., Gallagher, J. S., Rice, W. L., \& Low, F. J. 1986, ApJ, 303,171

Hauser, M. G., Arendt, R. G., Kelsall, T., et al. 1998, ApJ, 508, 25

Kalberla, P. M. W., Burton, W. B., \& Hartmann, Dap. 2005, A\&A, 440, 775

Kennicutt, R. C. Jr. 1998a, ApJ, 498, 541

Kennicutt, R. C. Jr. 1998b, ARA\&A, 36, 189

Kent, S. M., Dame, T. M., \& Fazio, G. 1991, ApJ, 378, 131

Kelsall, T., Weiland, J. L., Franz, B. A., et al. 1998, ApJ, 508, 44

Kewley, L. J., Geller, M. J., Jansen, R. A., \& Dopita, M. A. 2002, AJ, 124, 3135

Kylafis, N. D., \& Bahcall, J. N. 1987, ApJ, 317, 637

Kylafis, N. D., \& Misiriotis, A. 2005 [arXiv: astro-ph/0501373]

Lehnert, M. D., \& Heckman, T. M. 1996, ApJ, 472, 546

Lyne, A. G., Manchester, R. N., \& Taylor, J. H. 1985, MNRAS, 213, 613

Mather, J. C., Johnson, N. J. E., Wright, E. L., \& Shoemaker, D. H. 1982, BAAS, 14,941

Misiriotis, A., Popescu, C. C., Tuffs, R. J., \& Kylafis, N. D. 2001, A\&A, 372, 775

Misiriotis, A., Papadakis, I. E., Kylafis, N. D., \& Papamastorakis, J. 2004, A\&A, 417,39

Meurer, G. R., Heckman, T. M., Lehnert, M. D., Leitherer, C., \& Lowenthal, J. 1997, AJ, 114, 54

Popescu, C. C., Misiriotis, A., Kylafis, N. D., Tuffs, R. J., \& Fischera, J. 2000, A\&A, 362, 138

Reach, W. T., Dwek, E., Fixsen, D. J., et al. 1995, ApJ, 451, 188

Schlegel, D. J., Finkbeiner, D. P., \& Davis, M. 1998, ApJ, 500, 525

Schmidt, M. 1959, ApJ, 129, 243

Snyder, J. P. 1987, U. S. Geological Survey, Washington, DC: U. S. Government Printing Office, 1395

Sodroski, T. J., Bennett, C., Boggess, N., et al. 1994, ApJ, 428, 638

van den Bosch, F. C., Robertson, B. E., Dalcanton, J. J., \& de Blok, W. J. G. 2000, AJ, 119, 1579

Weingartner, J. C., \& Draine, B. T. 2001, ApJ, 548, 296

Xilouris, E. M., Kylafis, N. D., Papamastorakis, J., Paleologou, E. V., \& Haerendel, G. 1997, A\&A, 325, 135

Xilouris, E. M., Alton, P. B., Davies, J. I., et al. 1998, A\&A, 331, 894

Xilouris, E. M., Byun, Y. I., Kylafis, N. D., Paleologou, E. V., \& Papamastorakis, J. 1999, A\&A, 344, 868 